\title{
Semiconducting (Half-Metallic) Ferromagnetism in Mn(Fe) Substituted Pt and Pd Nitrides
}

\author{
Abdesalem Houar:* \\ Laboratoire de Physique Théorique, Département de Physique, Université de Béjaia, Béjaia, Algérie \\ Samir F. Matar \\ CNRS, Université de Bordeaux, ICMCB, 87 avenue du Docteur Albert Schweitzer, 33600 Pessac, France \\ Volker Eyerti \\ Center for Electronic Correlations and Magnetism, \\ Institut für Physik, Universität Augsburg, 86135 Augsburg, Germany
}

(Dated: May 28, 2022)

\begin{abstract}
Using first principles calculations as based on density functional theory, we propose a class of so far unexplored diluted ferromagnetic semiconductors and half-metals. Here, we study the electronic properties of recently synthesized $4 d$ and $5 d$ transition metal dinitrides. In particular, we address $\mathrm{Mn}-$ and Fe-substitution in $\mathrm{PtN}_{2}$ and $\mathrm{PdN}_{2}$. Structural relaxation shows that the resulting ordered compounds, $\mathrm{Pt}_{0.75}(\mathrm{Mn}, \mathrm{Fe})_{0.25} \mathrm{~N}_{2}$ and $\mathrm{Pd}_{0.75}(\mathrm{Mn}, \mathrm{Fe})_{0.25} \mathrm{~N}_{2}$, maintain the cubic crystal symmetry of the parent compounds. On substitution, all compounds exhibit long-range ferromagnetic order. While both $\mathrm{Pt}_{0.75} \mathrm{Mn}_{0.25} \mathrm{~N}_{2}$ and $\mathrm{Pd}_{0.75} \mathrm{Mn}_{0.25} \mathrm{~N}_{2}$ are semiconducting, Fe-substitution causes halfmetallic behavior for both parent materials.

PACS numbers: 71.15.Mb, 71.15.Nc, 71.20-b, 75.10.Lp, 74.25.Ha, 73.43.Cd

Keywords:
\end{abstract}

Being known since the beginning of the 20th century, transition metal nitrides are considered as an exciting class of materials due to a wide range of technological applications. Traditionally, the great advantages of these compounds concern their hardness and refractory nature 1.2 . However, much attention is currently directed towards their electronic, magnetic, and optical properties, where fascinating applications are expected. Many efforts, experimental as well as theoretical, have been made to study the transition metal nitrides ${ }^{3}-\underline{6}$. Until recently none of the noble metal nitrides or the platinum group nitrides were known. The first synthesis of platinum nitride $(\mathrm{Pt}-\mathrm{N})$, under extreme conditions of pressure and temperature, was reported only few years ago $\frac{7.8}{}$. Lateron, several other nitrides of different elements (Ir, Os, $\mathrm{Ru}$ and $\mathrm{Pd}$ ) were also obtained ${ }^{9}-11$.

There was a debate about the crystal structure and the stoichiometry of platinum nitride. While a zinc-blende structure was first proposed, Crowhurst et al. demonstrated that this nitride crystallizes neither in zinc-blende (PtN: mononitride) nor in fluorite $\left(\mathrm{PtN}_{2}\right.$ : dinitride) type structures, which are highly unstable at the synthesis conditions $(P=50 \mathrm{GPa}$ and $T=2000 \mathrm{~K}) \underline{\underline{8}}$. Instead, the authors revealed that the coumpound is a dinitride, hence $\mathrm{PtN}_{2}$, and the ground state structure is a cubic pyrite structure. Lateron, these authors succeeded in synthesizing $\operatorname{IrN}_{2}$ and $\mathrm{PdN}_{2}$, where the first one is found to be in the monoclinic baddeleyite structure ${ }^{9}$. Yet, $\mathrm{PdN}_{2}$, which could by synthesized at high pressures but was not stable at ambient conditions, was proposed to also crystallize in the pyrite structure. In a recent theoretical investigation it was shown that tetragonal distortions may stabilize $\mathrm{PdN}_{2}$ at ambient pressure $\frac{12}{2}$. Other nitrides
$\left(\mathrm{OsN}_{2}, \mathrm{RuN}_{2}\right.$ and $\left.\mathrm{RhN}_{2}\right)$ have been also obtained and are shown to crystallize in marcasite type structure 10,11 .

Platinum dinitride has been predicted to have excellent mechanical properties. The calculated hardness (bulk modulus, shear modulus and other elastic constants) shows that it is harder than many known hard materials like $\mathrm{TiN}$ and $\mathrm{SiC}^{13}, 14$. The electronic properties of $\mathrm{PtN}_{2}$ are also very interesting. Contrary to other transition metal nitrides, which are almost all metallic, $\mathrm{PtN}_{2}$ is found to be semiconducting, and this could make it an important material for optoelectronic applications. Band structure calculations as based on density functional theory and the local density approximation (LDA) show an indirect band gap of $\sim 1.5 \mathrm{eV}$, which is probably somewhat smaller than the experimental value due to the tendency of the LDA to underestimate the band gap $13-15$.

In general, pyrite-type compounds have attracted attention since long. The dinitrides $\mathrm{AN}_{2}(\mathrm{~A}=\mathrm{C}, \mathrm{Si}, \mathrm{Ge})$ were devised in assumed pyrite-type structures leading to compounds with peculiar properties, such as the extreme hardness obtained for $\mathrm{CN}_{2}$ with a bulk modulus of $405 \mathrm{GPa}$. For these systems characterized as wide band gap semiconductors, strong hybridization of the $\mathrm{N} 2 p$ states with the A $p$ states results in a depression of the optical band gap along the C, Si, Ge series16.17. Pyrite-type disulfides have also been of considerable interest for different reasons $\frac{18}{2}$. Semiconducting $\mathrm{FeS}_{2}$ has found widespread attention for its application in photovoltaic energy conversion ${ }^{19}$. $\mathrm{ZnS}_{2}$ is a diamagnetic insulator. Substitution of $\mathrm{Zn}$ for Fe in iron pyrite has thus been used to tune the optical band gap in order to enhance the response to the solar spectrum 20 . While $\mathrm{FeS}_{2}$ is a van Vleck paramagnet, metallic $\mathrm{CoS}_{2}$ displays long- 
range ferromagnetic order. In contrast, $\mathrm{NiS}_{2}$ is an antiferromagnetic insulator, where the insulating behaviour has been attributed to the presence of strong electronic correlations.

Our present work is focused especially on the electronic and magnetic properties of substituted $\mathrm{PtN}_{2}$ and $\mathrm{PdN}_{2}$. We demonstrate that substitution of the non-magnetic $4 d$ - and $5 d$-transition metal ions by the magnetic $3 d$-ions $\mathrm{Mn}$ and Fe may lead to semiconducting and half-metallic ferromagnetism, respectively.

In our investigation, we first consider Mn-substitution in $\mathrm{PtN}_{2}$ and $\mathrm{PdN}_{2}$. For the latter compound, we assumed a cubic pyrite crystal structure as for $\mathrm{PtN}_{2}$. This assumption is based on the fact that experimentally $\mathrm{PdN}_{2}$ is actually synthesized in cubic pyrite structure at high pressure conditions, even though it is not stable at ambient pressure ${ }^{9}$. Replacing one of the four $\mathrm{Pt}$ and $\mathrm{Pd}$ by magnetic $\mathrm{Mn}$ leads to $\mathrm{Pt}_{0.75} \mathrm{Mn}_{0.25} \mathrm{~N}_{2}$ and $\mathrm{Pd}_{0.75} \mathrm{Mn}_{0.25} \mathrm{~N}_{2}$, respectively. To check if the cubic symmetry is maintained on Mn-substitution, a quantum molecular dynamics relaxation has been performed using the Siesta $a b$ initio simulation package with normconserving pseudopotentials 21,22 . Both atomic positions and cell shape were included in the relaxation process. As a result, neither $\mathrm{Pt}_{0.75} \mathrm{Mn}_{0.25} \mathrm{~N}_{2}$ nor $\mathrm{Pd}_{0.75} \mathrm{Mn}_{0.25} \mathrm{~N}_{2}$ display any deviations from cubic symmetry and the atoms remain nearly at the positions of the pure compound. In particular, the internal nitrogen parameters are almost unchanged after Mn-substitution in both $\mathrm{PtN}_{2}$ and $\mathrm{PdN}_{2}$. The changes in the nitrogen positions are within $0.07 \AA$. To be specific, in $\mathrm{PtN}_{2}$ the internal nitrogen parameter changes from 0.415 (as given in Ref. 8) to 0.416 after the relaxation of the substituted system.

In a second step, full potential augmented spherical wave (FPASW) calculations were carried out $\underline{23}, 24$ in order to address the electronic properties of all compounds under study. To start with, we recalculated the equilibrium lattice constant. From non-spin polarized LDA calculations, we obtained a lattice parameter of $a_{N M}=4.79 \AA$ for $\mathrm{Pt}_{0.75} \mathrm{Mn}_{0.25} \mathrm{~N}_{2}$. Taking into account spin polarization led to a slightly larger value of $a_{F M}=4.82 \AA$, with the ferromagnetic state being more stable than the nonmagnetic one. It is important to note that the values obtained for the lattice constant of $\mathrm{Pt}_{0.75} \mathrm{Mn}_{0.25} \mathrm{~N}_{2}$ resemble that of $\mathrm{PtN}_{2}$, which is $a=4.80 \AA$, and confirm the molecular dynamics result. To conclude from both sets of calculations, not only the cubic symmetry is preserved after Mn-substitution, but even the lattice constant is almost not affected. The negligible changes of the structure can be understood from the fact that only one out of four Pt atoms is replaced and thus the platinum network is affected by the substitution only to a small degree. Motivated by these findings, we decided to perform the calculations for $\mathrm{Pd}_{0.75} \mathrm{Mn}_{0.25} \mathrm{~N}_{2}$ using the same lattice constant as for $\mathrm{PdN}_{2}$, i.e. $a=4.75 \AA$ (see also the discussion below).

Subsequently, the electronic structures of the Mnsubstituted compounds were analyzed in terms of the projected densities of states as arising from the FPASW calculations. However, for the Mn-substituted systems the LDA results bear some ambiguity. To be specific, we obtain semiconducting behavior for $\operatorname{Pd}_{0.75} \mathrm{Mn}_{0.25} \mathrm{~N}_{2}$, whereas $\mathrm{Pt}_{0.75} \mathrm{Mn}_{0.25} \mathrm{~N}_{2}$ is at the verge of being a semiconductor but displays a small band overlap. In order to check these findings, we additionally performed a set of calculations based on the GGA ${ }^{25}$. They resulted in semiconducting ferromagnetic ground states for both compounds with indirect band gaps of $0.17 \mathrm{eV}$ and $0.42 \mathrm{eV}$ for $\mathrm{Pt}_{0.75} \mathrm{Mn}_{0.25} \mathrm{~N}_{2}$ and $\mathrm{Pd}_{0.75} \mathrm{Mn}_{0.25} \mathrm{~N}_{2}$, respectively. The corresponding partial densities of states (DOS) are illustrated in Figs. 1 and 2 The spectrum falls essentially

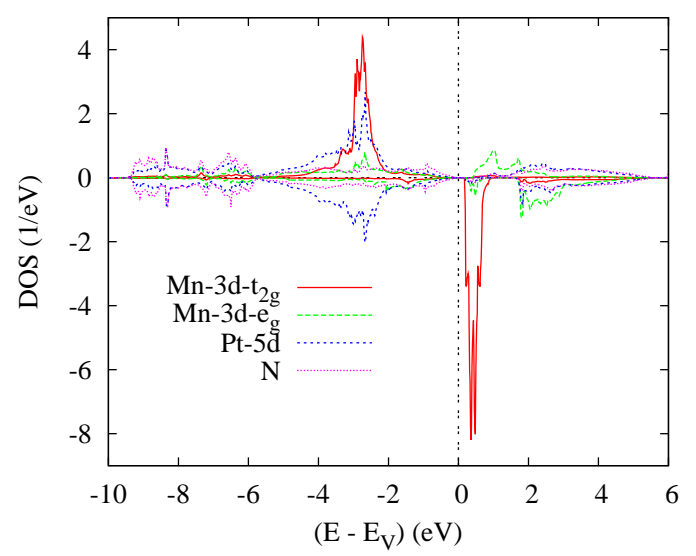

FIG. 1: (Color online) Partial DOS of $\mathrm{Pt}_{0.75} \mathrm{Mn}_{0.25} \mathrm{~N}_{2}$. Here and in all subsequent figures, $t_{2 g}$ and $e_{g}$ orbitals are referred to a rotated coordinate system with the Cartesian axes pointing along the metal-nitrogen bonds.

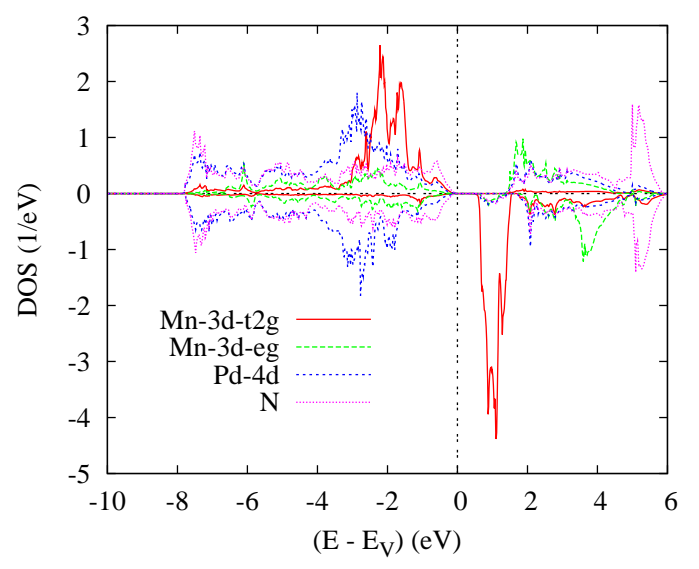

FIG. 2: (Color online) Partial DOS of $\mathrm{Pd}_{0.75} \mathrm{Mn}_{0.25} \mathrm{~N}_{2}$.

in four parts. While the low-energy range from -9 to $-6 \mathrm{eV}$ and from -8 to $-4 \mathrm{eV}$ for $\mathrm{Pt}_{0.75} \mathrm{Mn}_{0.25} \mathrm{~N}_{2}$ and $\mathrm{Pd}_{0.75} \mathrm{Mn}_{0.25} \mathrm{~N}_{2}$, respectively, is dominated by the $\mathrm{N} 2 p$ states, the upper valence band is formed mainly by the $t_{2 g}$ manifolds of the transition metal $d$ states. For the 
spin-minority bands, the situation is slightly more complicated. Whereas the Pd $4 d$ and Pt $5 d$ states are fully occupied and found in the same energy range as their spinmajority counterparts, the Mn $3 d t_{2 g}$ states experience strong exchange splitting. As a result, the Mn $3 d t_{2 g}$ spindown states form the lower conduction band of this spin channel and a magnetic moment of $3 \mu_{B}$ is found at these atoms. In contrast, spin polarizations of $\mathrm{Pd}, \mathrm{Pt}$, and $\mathrm{N}$ are negligible. Finally, the remaining conduction band states can be attributed to the transition metal $d$ states of $e_{g}$ symmetry. Since the latter form $\sigma$-type bonds with the N $2 p$ states, we find a considerable admixture of both types of states in the lower valence and upper conduction band. This admixture is much smaller for the bands between -6 and $+1 \mathrm{eV}$ and -4 to $+2 \mathrm{eV}$, respectively, which are of $t_{2 g}$ symmetry and form less strong $\pi$ bonds. In passing we mention the albeit small band gaps, which make both Mn-substituted compounds semiconducting. Yet, we note that LDA and GGA underestimate the optical band gap, which might thus be considerably larger in reality. In order to check this, we performed additional GGA $+U$ calculations for $\mathrm{Pt}_{0.75} \mathrm{Mn}_{0.25} \mathrm{~N}_{2}$. While there were no qualitative changes, both the exchange splitting of the Mn $3 d t_{2 g}$ states and the optical band gap increased considerably.

The second substitution that we considered was the replacement of $\mathrm{Pt}$ and $\mathrm{Pd}$ by iron, leading to the ordered compounds $\mathrm{Pt}_{0.75} \mathrm{Fe}_{0.25} \mathrm{~N}_{2}$ and $\mathrm{Pd}_{0.75} \mathrm{Fe}_{0.25} \mathrm{~N}_{2}$. Our procedure was the same as for Mn-substitution. Molecular dynamics relaxations using the Siesta code (with the same calculations details cited above)w were performed including relaxation of both the atomic positions and the cell shape. As in the Mn-case we found that the cubic symmetry is not broken on Fe-substitution and that even the internal nitrogen parameter remained essentially unchanged.

For the FPASW calculations performed in a second step in order to address the electronic and magnetic properties, we followed the procedure already adopted for $\mathrm{PdN}_{2}$ and used the lattice constants of the pure systems also for the substituted materials. In this case, our procedure was justified by an additional recalculation of the equilibrium lattice constant for $\mathrm{Pd}_{0.75} \mathrm{Fe}_{0.25} \mathrm{~N}_{2}$. As a result, values of $a_{N M}=4.742 \AA$ and $a_{F M}=4.749 \AA$ were obtained as arising from non-spin polarized and spin polarized calculations, respectively. The latter value is almost identical to the value of $a=4.75 \AA$ of pure $\mathrm{PdN}_{2}$.

Again, the LDA results bear some ambiguity as they led to half-metallic behavior for $\operatorname{Pd}_{0.75} \mathrm{Fe}_{0.25} \mathrm{~N}_{2}$ but metallicity of both spin channels for $\mathrm{Pt}_{0.75} \mathrm{Fe}_{0.25} \mathrm{~N}_{2}$. Yet, the spin-majority density of states at the Fermi energy was found to be very small. The problem could again be resolved by GGA calculations, which render both substituted materials half-metallic. Both compounds exhibit stable magnetic order with magnetic moments of $2.0 \mu_{B}$ located almost completely at the iron atoms.

The electronic structure and partial DOS of the two compounds as arising from the spin-polarized ferromag- netic calculations are illustrated in Figs. 3 and 4 The

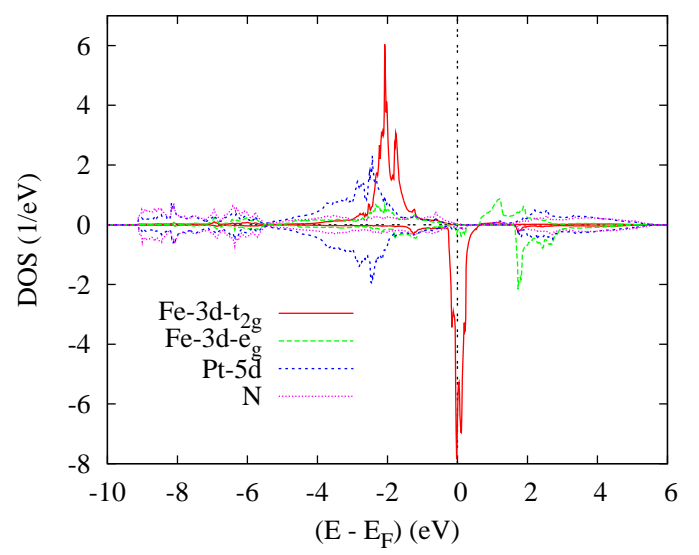

FIG. 3: (Color online) Partial DOS of $\mathrm{Pt}_{0.75} \mathrm{Fe}_{0.25} \mathrm{~N}_{2}$.

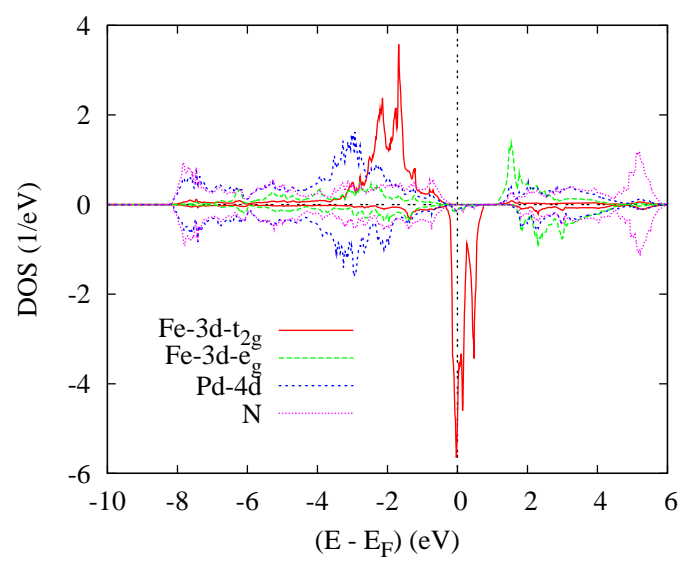

FIG. 4: (Color online) Partial DOS of $\mathrm{Pd}_{0.75} \mathrm{Fe}_{0.25} \mathrm{~N}_{2}$.

gross features of the partial densities of states are the same as for the Mn-substituted compounds. Differences are due to the smaller magnetic moments of the Fe atoms, which lead to reduced exchange splittings of the $3 d t_{2 g}$ states. As a consequence, the respective spin-majority bands are shifted to higher energies as compared to the $d$ states of the $\mathrm{Pt}$ and $\mathrm{Pd}$ matrix. In addition, the $\mathrm{Fe}$ spin-minority bands are shifted to lower energies as compared to the Mn-systems due to the increased electron count. As a result, the semiconducting behavior is lost and a metallic spin-down channel found. Again, these results were qualitatively confirmed by additional GGA $+U$ calculations, which revealed an increase of the exchange splitting of the Fe $3 d t_{2 g}$ states as well as the band gap of the spin-majority channel.

In passing, we mention additional spin polarized calculations, which were performed $\mathrm{Pt}_{0.75} \mathrm{Mn}_{0.25} \mathrm{~N}_{2}$ in order to check for long range antiferromagnetic order. For these calculations, we used a tetragonal structure arising from 
doubling the cubic cell along the $c$ axis. As a result, an antiferromagnetic and again semiconducting solution was found albeit with a total energy, which by about 7 mRy/f.u. higher than that of the ferromagnetic ground state.

In summary, based on our first principles investigation we propose the existence of so far unexplored diluted ferromagnetic semiconductors and half-metals. These materials arise from substituting magnetic $3 d$ ions (Mn, Fe) in the non-magnetic dinitrides $\mathrm{PtN}_{2}$ and $\mathrm{PdN}_{2}$. According to molecular dynamics calculations, the ordered compounds $\mathrm{A}_{0.75} \mathrm{~B}_{0.25} \mathrm{~N}_{2}$, where $\mathrm{A}=\mathrm{Pt}, \mathrm{Pd}$ and $\mathrm{B}=\mathrm{Mn}, \mathrm{Fe}$, preserve the cubic pyrite structure of their parent compounds. On substitution, stable long-range ferromag- netic order results with magnetic moments of $3 \mu_{B}$ and $2 \mu_{B}$, which are well localized at the Mn- and Fe-sites, respectievly. While Mn-substitution leads to semiconducting behavior, introduction of iron causes the substituted compounds to be half-metallic. Our results still await experimental confirmation.

\section{Acknowledgments}

This work was supported by the Deutsche Forschungsgemeinschaft through TRR 80 .
* Corresponding authors: habdslam@yahoo.fr

† volker@eyert.de

1 S. T. Oyama, Introduction to the chemistry of transition metal carbides and nitrides, in: The chemistry of Transition Metal Carbides and Nitrides, edited by S. T. Oyama (Blackie Academic \& Professional, London 1996), Chap. 1.

2 H. O. Pierson, Handbook of Refractory Carbides and $\mathrm{Ni}$ trides (Noyes Publications, Westwood 1996), Chaps. 9-13.

3 A. Houari, S. F. Matar, M. A. Belkhir, and M. Nakhl, Phys. Rev. B 75, 064420 (2007).

4 A. Houari, S. F. Matar, and M. A. Belkhir, Computational. Material. Science 43, 392 (2008).

5 S. Jhi, J. Ihm, S. G. Louis, and M. L. Cohen, Nature 399, 132 (1999).

6 U. Lundin, L. Fast, L. Nordstrom, B. Johansson, J. M. Wills, and O. Eriksson, Phys. Rev. B 57, 4979 (1998).

7 E. Gregoryanz, C. Sanloup, M. Somayazulu, J. Badro, G. Fiquet, H. K. Mao, and R. J. Hemley, Nat. Mater. 3, 294 (2004).

8 J. C. Crowhurst, A. F. Goncharov, B. Sadigh, C. L. Evans, P. G. Morrall, J. L. Ferreira, and A. J. Nelson, Science 311, 1275 (2006).

9 J. C. Crowhurst A. F. Goncharov, B. Sadigh, J. M. Zaug, D. Aberg, Y. Meng, V. B. Prakapenka, J. Mater. Res. 23, 1 (2008).

10 A. F. Young, C. Sanloup, E. Gregoryanz, S. Scandolo, R. J. Hemley, and H. K. Mao, Phys. Rev. Lett. 96, 155501 (2006).

11 R. Yu, Q. Zhan, and L. C. De Jonghe, In- gew. Chem. Int. Ed. 46, 1136 (2007).

12 D. Aberg, B. Sadigh, J. C. Crowhurst and A. F. Goncharov, Phys. Rev. Lett. 100, 095501 (2008).

13 R. Yu, Q. Zhan and X. F. Zhang, Appl. Phys. Lett. 88, 051913 (2006).

14 H. Gou, L. Hou, J. Zhang, G. Sun, L. Gao and F. Gao, Appl. Phys. Lett. 89, 141910 (2006).

15 A. F. Young, J.A. Montoya, C. Sanloup, M. Lazzeri, E. Gregoryanz, and S. Scandolo, Phys. Rev. B 73, 153102 (2006).

16 R. Weihrich, S. F. Matar, E. Betranhandy, and V. Eyert, Solid State Sciences 5, 701 (2003).

17 R. Weihrich, V. Eyert, and S. F. Matar, Chem. Phys. Lett. 373, 636 (2003).

18 J. B. Goodenough, J. Solid State Chem. 3, 26 (1971),

19 V. Eyert, K.-H. Höck, S. Fiechter, and H. Tributsch, Phys. Rev. B 57, 6350 (1998).

${ }^{20}$ K. Büker, S. Fiechter, V. Eyert, and H. Tributsch, J. Electrochem. Soc. 146, 261 (1999).

21 J. M. Soler, J. D. Gale, A. Garcia, J. Junquera, P. Ordejon, D. Sanchez-Portal, J. Phys: Cond. Matter 14, 2747 (2002).

22 N. Troullier and J. L. Martins, Phys. Rev. B 43, 1993 (1991).

23 V. Eyert, Int. J. Quantum Chem. 77, 1007 (2000).

${ }^{24}$ V. Eyert, The Augmented Spherical Wave Method A Comprehensive Treatment, Lect. Notes Phys. 719 (Springer, Heidelberg, 2007).

25 J. P. Perdew, K. Burke, and M. Ernzerhof, Phys. Rev. Lett. 77, 3865 (1996). 